\title{
Glucose Monitoring During Pregnancy
}

\author{
J. Seth Hawkins
}

Published online: 30 March 2010

(C) The Author(s) 2010. This article is published with open access at Springerlink.com

\begin{abstract}
Self-monitoring of blood glucose in women with mild gestational diabetes has recently been proven to be useful in reducing the rates of fetal overgrowth and gestational weight gain. However, uncertainty remains with respect to the optimal frequency and timing of selfmonitoring. A continuous glucose monitoring system may have utility in pregnant women with insulin-treated diabetes, especially for those women with blood sugars that are difficult to control or who experience nocturnal hypoglycemia; however, continuous glucose monitoring systems need additional study as part of larger, randomized trials.
\end{abstract}

Keywords Diabetes - Gestational diabetes - Glucose monitoring $\cdot$ Self-monitoring of blood glucose $\cdot$ SMBG

\section{Introduction}

An association between diabetes in pregnancy and fetal overgrowth has long been recognized. Fetal overgrowth is associated with a number of adverse outcomes for both the mother and her baby, such as a higher rate of difficult delivery. To reduce the rate of fetal overgrowth and its associated complications, women with diabetes in pregnancy undergo a number of interventions. Among these interventions is glucose monitoring. Although glucose monitoring during pregnancies complicated by diabetes

\footnotetext{
J. S. Hawkins $(\bowtie)$

Department of Obstetrics \& Gynecology,

University of California, Irvine,

101 The City Drive South, Building 56, Suite 800,

Orange, CA 92868, USA

e-mail: jshawkin@uci.edu
}

mellitus is widely practiced, it is important to ask a number of questions:

1. Is there evidence that glucose monitoring improves outcomes in women with gestational diabetes?

2. Which glycemic parameters are associated with adverse pregnancy outcomes and are therefore best used in management?

3. What cutoffs for normal and abnormal glucose values are appropriate in a glucose monitoring regimen?

4. What is the utility of measuring glycosylated hemoglobin during gestation?

5. Is a continuous glucose monitoring system (CGMS) beneficial?

In answering these questions, this article focuses on recent articles that appeared between 2007 and 2009. A PubMed search using the terms "diabetes" and "pregnancy" was used to select relevant studies.

\section{Is There Evidence That Glucose Monitoring Improves Outcomes in Women With Gestational Diabetes?}

With the advent of home reflectance monitors in the late 1970s and early 1980s, self-monitoring of blood glucose (SMBG) was demonstrated to be possible, and perhaps beneficial, in women with gestational diabetes $[1,2]$. By 2004, SMBG was widely practiced, with more than $90 \%$ of obstetrician/gynecologists recommending that their patients with gestational diabetes check fasting blood glucose (FBG). However, only $61 \%$ of obstetrician/gynecologists were recommending 2-hour postprandial tests [3]. This lack of uniformity in the approach to glucose monitoring can be attributed to several factors. Specifically, there has been uncertainty about the frequency and utility of daily testing; 
the necessity of SMBG in all women with diabetes during pregnancy; the timing of self-monitoring measurements and whether preprandial or postprandial testing was superior; and the ideal thresholds for implementing changes in therapy [4].

With respect to the uncertain benefits of SMBG in women with diet-treated gestational diabetes, we recently published our findings regarding the utility of SMBG at the University of Texas Southwestern Medical Center [5]. Between January 1991 and December 1997, women with diet-treated gestational diabetes had routine glycemic monitoring at weekly prenatal visits [5]. After January 1998, the standard practice was to issue home glucose meters with instructions to check blood glucose levels four times per day. A total of 675 women who underwent weekly monitoring were compared with 315 women who performed SMBG four times per day. We found that SMBG was associated with a number of maternal and fetal benefits. In particular, there were significantly fewer overgrown infants, with rates of macrosomic and largefor-gestational-age infants at $21.9 \%$ and $23.1 \%$, respectively, among women using SMBG, compared with $29.9 \%$ and $34.4 \%$, respectively, for women monitored weekly ( $P$ values, 0.013 and $\leq 0.001$, respectively). Women using SMBG gained significantly less weight when compared with women monitored weekly. Women using selfmonitoring gained a median of $0.56 \mathrm{lb}$ per week (interquartile range, 0.22-1.08) compared with $0.74 \mathrm{lb}$ per week (interquartile range, $0.33-1.17 ; P=0.009$ ) when compared with women monitored weekly. Given that there is an association between excessive gestational weight gain and postpartum weight retention [6] with all of its potential long-term sequelae, it seems prudent to incorporate pregnancy interventions that limit weight gain.

These data are supported by the recent findings of Wilson et al. [7], who compared two cohorts composed of 50 randomly selected patients from the 2000-2002 and 20062008 epochs. The intention of the authors was to assess the effect of three interventions incorporated into routine prenatal care for women with diet-treated gestational diabetes at their institution between 2003 and 2005. These three interventions included dietary advice, regular contact with a diabetic team, and 1-hour postprandial SMBG (with a glycemic target $\leq 7.8 \mathrm{mmol} / \mathrm{L}$ ). Although it may be difficult to separate the effect of dietary advice and contact with the diabetic team from the effect of glucose monitoring, the cumulative benefit was a reduction in neonatal birth weights for women who received these interventions, including SMBG (3,269 vs. $3,567 \mathrm{~g} ; P=0.033)$.

Recent expert opinion also supports the utility of SMBG in women with gestational diabetes. Writing in Endocrine Practice, Dr. Jovanovic [8] emphasized the importance of a self-monitoring regimen incorporating postprandial glucose determination because postprandial hyperglycemia has been correlated with fetal overgrowth. Dr. Jovanovic advocates an intensive approach and recommends self-monitoring four times per day in patients with diet-treated gestational diabetes, and six times per day in women with insulintreated gestational diabetes. Other experts also emphasize the importance of SMBG by women with gestational diabetes [9] and pregestational diabetes [10].

\section{Which Glycemic Parameters Are Associated With Adverse Pregnancy Outcomes and Therefore Are Best Used in Management?}

Several glycemic parameters can be considered for glucose monitoring in women with gestational diabetes. The recent evidence for fasting, postprandial, and preprandial glucose values are each discussed in turn.

Fasting blood sugar is a valuable glycemic parameter for assessing the risk of undesirable pregnancy outcomes. Recently, Gonzalez-Quintero et al. [11] compared 2,030 women with gestational diabetes and good glucose control to 1,188 women with gestational diabetes but with poor glucose control. The percentage of patients with the composite outcome (defined as one or more of the following: macrosomia, large for gestational age, stillbirth, neonatal hypoglycemia, or jaundice) more than doubled $(57.9 \%$ vs. $24.8 \%)$ with an FBG of at least $123.5 \mathrm{mg} / \mathrm{dL}$, compared with FBG levels less than $95 \mathrm{mg} / \mathrm{dL}$. Voldner et al. [12] also found that fasting plasma glucose is a significant determinant of macrosomia (OR, 1.9; $95 \% \mathrm{CI}$, 1.1-3.4), especially for women with a body mass index of at least $27 \mathrm{~kg} / \mathrm{m}^{2}$. Such women had an OR of 4.5 (95\% CI, $1.7-12.5)$ for neonates with macrosomia.

These latter findings are consistent with the results of several recent studies assessing the effect of FBG measured during initial glucose tolerance testing. Seshiah et al. [13] screened 12,056 consecutive women and reported that the risk of delivering a large-for-gestational-age neonate rose with FBG levels above $80 \mathrm{mg} / \mathrm{dL}$, and was significantly increased above $90 \mathrm{mg} / \mathrm{dL}$. Additionally, Riskin-Mashiah et al. [14] studied 6,129 women who underwent glucose tolerance testing in the first trimester, but who did not have pregestational diabetes or FBG values greater than $105 \mathrm{mg} / \mathrm{dL}$. There was an increasing risk of large-for-gestational-age or macrosomic infants with rising FBG values above $75 \mathrm{mg} / \mathrm{dL}$, and these risks were significant above $80 \mathrm{mg} / \mathrm{dL}$. Likewise, the HAPO (Hyperglycemia and Adverse Pregnancy Outcomes) trial [15*0] of 23,316 women reported the blinded results of 75-g glucose tolerance testing performed from 24 to 32 weeks' gestation. This landmark study showed that there were continuous associations between increasing maternal glucose levels (fasting as well as at 1- and 2-hours 
postprandial) and birth weight. However, the reference group was composed of women with fasting glucose values less than $75 \mathrm{mg} / \mathrm{dL}$, a measurement substantially less than the present threshold for diagnosis of gestational diabetes. As the accompanying editorial for the HAPO trial noted, it is questionable whether lowering the threshold for the diagnosis and treatment of gestational diabetes would confer a benefit, without also potentially increasing the risk of poor fetal growth [16].

Recent evidence supports the contention by prominent investigators that postprandial hyperglycemia is a critical determinant in the development of the oversized fetus [8], but these recent data also suggest that preprandial hyperglycemia is similarly a factor in fetal overgrowth. Herranz et al. [17] studied 73 women with type 1 diabetes mellitus in pregnancy who were monitored six times daily during preconception and throughout pregnancy. Postprandial hyperglycemia, as well as preprandial hyperglycemia, were noted to be important variables in determining fetal size, with adjusted ORs of 2.03 (95\% CI, 1.14-3.60) and 2.97 (95\% CI, 1.31-6.73), respectively. In another study of 70 women with type 1 diabetes mellitus, preprandial hyperglycemia was again noted to be an important variable in determining fetal size [18]. In particular, preprandial hyperglycemia between weeks 29 and 32 was a strong predictor of a large-for-gestational-age infant. It remains unproven whether the combination of both preprandial and postprandial monitoring is superior to either preprandial or postprandial monitoring alone in women with diabetes during pregnancy.

Although a number of different glucose parameters have been shown to be helpful in the glucose monitoring of women with gestational diabetes, recent evidence exists to support the practice of most investigators who check fasting and postprandial glucose values [3]. However, with respect to postprandial glucose monitoring, it remains unclear whether 2-hour postprandial values are superior to 1-hour postprandial glucose values.

\section{What Cutoffs for Normal and Abnormal Glucose Values Are Acceptable in a Glucose Monitoring Regimen?}

Dr. John B. O'Sullivan's pioneering work from the late 1950s through the 1970s demonstrated that gestational diabetes is associated with a number of adverse outcomes $[19,20]$. He also showed that intervening to reduce hyperglycemia could reduce the number of overgrown babies [21]. Dr. O'Sullivan established the criteria for the diagnosis of gestational diabetes by measuring glucose values using the Somogyi-Nelson method on whole venous blood in 752 pregnant women [22]. He then set an upward normal limit of less than two standard deviations from the mean for the fasting and 1,2, and 3-hour postprandial values [22]. In subsequent years, the method used to measure glucose changed from a venous blood-based system to a plasma-based system using glucose oxidase technology, resulting in values that were approximately $14 \%$ higher. Thus, in 1979, when the National Diabetes Data Group made recommendations for the diagnosis of gestational diabetes, the cutoff values that Dr. O'Sullivan had earlier reported were changed to $105,190,165$, and $145 \mathrm{mg} / \mathrm{dL}$ [23]. Subsequently, Carpenter and Coustan recommended lower values $(95,180,155$, and $140 \mathrm{mg} / \mathrm{dL})$ using a different algorithm to account for reducing substances [24].

The fasting value as recommended by the National Diabetes Data Group $(105 \mathrm{mg} / \mathrm{dL})$ and the fasting value as determined by Carpenter and Coustan $(95 \mathrm{mg} / \mathrm{dL})$ form the basis for the current recommended fasting values. Either value is acceptable for the diagnosis of gestational diabetes according to the most recent Practice Bulletin by the American College of Obstetricians and Gynecologists (ACOG) [4], although ACOG does suggest that a fasting value of $95 \mathrm{mg} / \mathrm{dL}$ be used as the criteria for initiating or changing insulin therapy in women with gestational diabetes [4], as well as pregestational diabetes [25]. For monitoring during labor and delivery, glucose is generally checked hourly, and insulin is started for a capillary blood glucose of $100 \mathrm{mg} / \mathrm{dL}$ or greater [26].

\section{What Is the Utility of Monitoring Glycosylated Hemoglobin During Gestation?}

The glycosylated hemoglobin concentration, also called hemoglobin $A_{1 c}$, reflects a longer duration of glycemic control, generally about 3 months, which approximates the life span of the red blood cell. The use of this glycemic parameter has not been as widely incorporated into the management of diabetes mellitus during pregnancy, because it does not reflect short-term glycemic variations that occur due to changes in diet or insulin therapy. However, it has been tested as a variable in recent studies for estimating risks of three separate adverse outcomes: congenital malformations, preterm birth, and overgrown infants. With respect to the risk of malformations, a recent review of seven prospective cohort studies by Guerin et al. [27] revealed an increasing risk of major and minor anomalies with rising hemoglobin $\mathrm{A}_{1 \mathrm{c}}$ concentrations. With respect to the risk of preterm birth, Ekbom et al. [28] reported rising rates of preterm birth according to hemoglobin $A_{1 c}$ measured at 28 weeks' gestation among 213 women with type 1 diabetes mellitus. In particular, the risk of spontaneous preterm birth increased substantially for every $1 \%$ rise in hemoglobin $A_{1 c}$, with an adjusted OR of $4.5(95 \%$ 
CI, 2.0-9.8). However, glycosylated hemoglobin values have shown less utility for assessing the risk of overgrown infants, as two recent reports indicate. First, Lapolla et al. [29] reported that hemoglobin $A_{1 c}$ was not a useful predictor of large-for-gestational-age or macrosomic newborns in their review of 611 women with hemoglobin $A_{1 c}$ measured between 24 weeks and 27 weeks of gestation. In another study, Kernaghan et al. [30] actually reported a negative correlation between hemoglobin $A_{1 c}$ and birth weight among 338 women with type 1 diabetes mellitus.

In summary, the utility of a hemoglobin $A_{1 c}$ value appears to be greatest when performed periconceptionally to estimate the risk of congenital anomalies, but it does not appear to confer substantial benefit for estimating the risk of fetal overgrowth or other adverse pregnancy outcomes.

\section{Is a Continuous Glucose-Monitoring System Beneficial?}

Continuous glucose monitoring in women with diabetes during pregnancy has provoked considerable recent interest, with no less than five clinical trials [31-35] and five reviews $[36,37,38 \cdot, 39,40]$ published since 2007 . This interest undoubtedly stems from the additional information that a CGMS provides. By taking readings throughout the day (every $10 \mathrm{~s}$, then averaging these readings over $5 \mathrm{~min}$ ), continuous glucose monitoring is thought to provide a more complete picture of interprandial glycemic variability. However, it has been unclear whether the 288 readings generated during a 24-hour period can improve clinical management. Moreover, continuous monitoring requires the uninterrupted insertion of a glucose oxidase tip into the subcutaneous tissue of the anterior abdominal wall to measure the glucose levels of interstitial fluid. Continuous glucose monitoring is thus considerably more invasive than traditional SMBG. The commentary by Charlton [41] details the practical aspects of how these devices function, as well as the perceived advantages and disadvantages of CGMS.

A recent pilot study was reported from an institution interested in the feasibility of continuous glucose monitoring. To assess the tolerability and utility of the Medtronic CGMS (Medtronic, Minneapolis, MN), McLachlan et al. [33] evaluated 55 women with diabetes during pregnancy. The majority of the participants were women with gestational diabetes ( 37 of 55), with the balance of patients divided between type $2(n=10)$ and type 1 diabetes $(n=8)$. Subjects who were assessed as having difficult glycemic control were issued a continuous glucose monitor, and most of these women completed at least $97 \%$ of the 72 -hour monitoring period. The objective of this monitoring was to obtain feedback from the perspective of both the patient and the treating clinician. In a follow-up feedback questionnaire, 37 of the 48 patients who responded (77\%) felt that the benefits of using a CGMS outweighed the risk, with $92 \%$ reporting that the device was "very easy" or "easy" to use and $81 \%$ reporting that the inconvenience was minimal or minor. Similarly, most $(62 \%)$ of the treating clinicians surveyed felt that the additional information was clinically useful and altered management, either by revealing overnight hypoglycemia (especially in type 1 diabetes) or by indicating the presence of hyperglycemia that was undetected by concurrent intermittent SMBG.

However, two recent comparative trials have not reported dramatic benefits for CGMS over SMBG. Both of these trials used an open-label, randomized, controlled design to directly compare CGMS with daily SMBG. In the first study by Kestila et al. [32], 36 women with gestational diabetes were randomized to CGMS (Medtronic) and 37 women to SMBG. Of these women, $31 \%$ in the CGMS group were ultimately prescribed insulin, metformin, or both, compared with only $8 \%$ of the SMBG group prescribed such therapy $(P=0.0149)$. Nonetheless, this additional antihyperglycemic therapy in the CGMS group did not translate into a benefit with respect to maternal or neonatal outcomes. In particular, there was no reduction in the rates of cesarean delivery or hypertension for women with gestational diabetes using the continuous monitoring system, nor was there any reduction in birth weight or rates of neonatal hypoglycemia. Apart from women with gestational diabetes, Murphy et al. [34] examined the effect of a CGMS on pregnant women with type 1 diabetes (46 women) or type 2 diabetes ( 25 women). These women were randomized to a CGMS (Medtronic) plus standard antenatal therapy (CGMS + SMBG, 38 women) or to standard antenatal therapy (SMBG, 33 women). There were more women with type 1 diabetes versus type 2 diabetes (74\% vs. 55\%) in the CGMS group, and this translated into a significantly greater mean duration of diabetes in the CGMS group ( 15.2 vs. 10.0 years; $P=0.03$ ). These women in the CGMS group ultimately delivered significantly smaller babies as measured by median birth weight centile (69th vs. 93rd; $P=0.02$ ), but the $P$ values for the rates of macrosomia ( $\geq 90$ th centile) and the mean birth weight standard deviation score were barely significant at 0.05 . Moreover, there were no significant differences in the rates of extremely large-for-gestational-age ( $\geq 97.7$ th centile) neonates, cesarean delivery, preeclampsia, or other measures of neonatal morbidity.

Two smaller, noncomparative studies assessed the effect of a CGMS during labor. Stenninger et al. [35] prospectively studied the use of a Medtronic CGMS in 15 women with insulin-treated diabetes during labor and 
delivery. The authors found that glucose levels in the $2 \mathrm{~h}$ prior to delivery correlated with the need for intravenous glucose therapy in the neonates, and they also reported that "the mothers coped well with the CGMS recording during labor." Similarly, Iafusco et al. [31] used a Medtronic CGMS during labor and delivery, as well as during betamethasone treatment for imminent preterm delivery in 18 women with type 1 diabetes. On the basis of this glycemic evaluation, decisions to begin insulin therapy were made, and the authors reported that were no cases of neonatal hypoglycemia or respiratory distress syndrome after delivery.

In summary, recent studies suggest that CGMS may be beneficial for certain women with diabetes treated with insulin, particularly in women with diabetes that is difficult to control. However, these data require further evaluation and do not yet support the incorporation of CGMS into routine practice. For an evaluation of all studies incorporating CGMS since 1999, the reader is directed to the comprehensive reviews by De Block et al. [38 $]$ and Block et al. [39].

\section{Conclusions}

Although of proven value in the management of women with overt diabetes, SMBG in women with mild gestational diabetes has only recently been proven to be useful in reducing the rates of fetal overgrowth and gestational weight gain. Yet, there remains uncertainty about the optimal frequency and timing of self-monitoring. The ACOG recommends that a value of $95 \mathrm{mg} / \mathrm{dL}$ be used as a threshold for fasting hyperglycemia $[4,25]$. The utility of testing glycosylated hemoglobin is limited to periconceptual measurements in evaluating the risk of congenital anomalies. A CGMS may have value in pregnant women with insulin-treated diabetes, especially for those whose blood sugars are difficult to control or may have nocturnal hypoglycemia, but this technology needs additional evaluation with larger, randomized, controlled trials.

Acknowledgments The author wishes to thank Dr. Brian Casey for his thoughtful suggestions. The author is supported by the Reproductive Scientist Development Program through both National Institutes of Health grant 2K12HD000849 and the March of Dimes Birth Defects Foundation (MOD).

Disclosure No potential conflict of interest relevant to this article was reported.

Open Access This article is distributed under the terms of the Creative Commons Attribution Noncommercial License which permits any noncommercial use, distribution, and reproduction in any medium, provided the original author(s) and source are credited.

\section{References}

Papers of particular interest, published recently, have been highlighted as:

- Of importance

•- Of major importance

1. Espersen T, Klebe JG: Self-monitoring of blood glucose in pregnant diabetics. Acta Obstet Gynecol Scand 1985, 64:11-14.

2. Landon MB, Cembrowski GS, Gabbe SG: Capillary blood glucose screening for gestational diabetes: a preliminary investigation. Am J Obstet Gynecol 1986, 155:717-721.

3. Gabbe SG, Gregory RP, Power ML, et al.: Management of diabetes mellitus by obstetrician-gynecologists. Obstet Gynecol 2004, 103:1229-1234.

4. American College of Obstetricians and Gynecologists Committee on Practice Bulletins-Obstetrics: ACOG Practice Bulletin. Clinical management guidelines for obstetrician-gynecologists. Number 30, September 2001 (replaces Technical Bulletin Number 200, December 1994). Gestational diabetes. (Reaffirmed 2008.) Obstet Gynecol 2001, 98:525-538.

5. Hawkins JS, Casey BM, Lo JY, et al.: Weekly compared with daily blood glucose monitoring in women with diet-treated gestational diabetes. Obstet Gynecol 2009, 113:1307-1312.

6. Siega-Riz AM, Viswanathan M, Moos MK, et al.: A systematic review of outcomes of maternal weight gain according to the Institute of Medicine recommendations: birthweight, fetal growth, and postpartum weight retention. Am J Obstet Gynecol 2009 , 201:339.e1-339.e14.

7. Wilson N, Ashawesh K, Kulambil Padinjakara RN, et al.: The multidisciplinary diabetes-endocrinology clinic and postprandial blood glucose monitoring in the management of gestational diabetes: impact on maternal and neonatal outcomes. Exp Clin Endocrinol Diabetes 2009, 117:486-489.

8. Jovanovic LG: Using meal-based self-monitoring of blood glucose as a tool to improve outcomes in pregnancy complicated by diabetes. Endocr Pract 2008, 14:239-247.

9. Cheng YW, Caughey, AB: Gestational diabetes: diagnosis and management. J Perinatol 2008, 28:657-664.

10. Gabbe SG, Carpenter LB, Garrison EA: New strategies for glucose control in patients with type 1 and type 2 diabetes mellitus in pregnancy. Clin Obstet Gynecol 2007, 50:1014-1024.

11. Gonzalez-Quintero VH, Istwan NB, Rhea DJ, et al.: The impact of glycemic control on neonatal outcome in singleton pregnancies complicated by gestational diabetes. Diabetes Care 2007, 30:467470 .

12. Voldner N, Qvigstad E, Froslie KF, et al.: Increased risk of macrosomia among overweight women with high gestational rise in fasting glucose. J Matern Fetal Neonatal Med 2010, 23:74-81.

13. Seshiah V, Balaji V, Panneerselvam A, et al.: "Abnormal" fasting plasma glucose during pregnancy. Diabetes Care 2008, 31:e92.

14. Riskin-Mashiah S, Younes G, Damti A, et al.: First trimester fasting hyperglycemia and adverse pregnancy outcomes. Diabetes Care 2009, 32:1639-1643.

15. • HAPO Study Cooperative Research Group, Metzger BE, Lowe LP, et al.: Hyperglycemia and adverse pregnancy outcomes. N Engl J Med 2008, 358:1991-2002. This is a large, prospective trial demonstrating the correlation between increasing hyperglycemia and increased rates of fetal overgrowth.

16. Ecker JL, Greene MF: Gestational diabetes-setting limits, exploring treatments. N Engl J Med 2008, 358:2061-2063.

17. Herranz L, Pallardo LF, Hillman N, et al.: Maternal third trimester hyperglycaemic excursions predict large-for-gestational-age 
infants in type 1 diabetic pregnancy. Diabetes Res Clin Pract 2007, 75:42-46.

18. Aschwald CL, Catanzaro RB, Weiss EP, et al.: Large-for-gestationalage infants of type 1 diabetic mothers: an effect of preprandial hyperglycemia? Gynecol Endocrinol 2009, 25:653-660.

19. Dandrow RV, O'Sullivan JB: Obstetric hazards of gestational diabetes. Am J Obstet Gynecol 1966, 96:1144-1147.

20. O'Sullivan JB, Charles D, Mahan CM, et al.: Gestational diabetes and perinatal mortality rate. Am J Obstet Gynecol 1973, 116:901904.

21. O'Sullivan JB, Gellis SS, Dandrow RV, et al.: The potential diabetic and her treatment in pregnancy. Obstet Gynecol 1966, 27:683-689.

22. Wilkerson HL, O'Sullivan JB: A study of glucose tolerance and screening criteria in 752 unselected pregnancies. Diabetes 1963, 12:313-318.

23. National Diabetes Data Group: Classification and diagnosis of diabetes mellitus and other categories of glucose intolerance. Diabetes 1979, 28:1039-1057.

24. Vidaeff AC, Yeomans ER, Ramin SM: Gestational diabetes: a field of controversy. Obstet Gynecol Surv 2003, 58:759-769.

25. ACOG Committee on Practice Bulletins: ACOG Practice Bulletin. Clinical Management Guidelines for Obstetrician-Gynecologists. Number 60, March 2005. Pregestational diabetes mellitus. (Reaffirmed 2007.) Obstet Gynecol 2005, 105:675-685.

26. Hawkins JS, Casey BM: Labor and delivery management for women with diabetes. Obstet Gynecol Clin North Am 2007, 34:323-334.

27. Guerin A, Nisenbaum R, Ray JG: Use of maternal GHb concentration to estimate the risk of congenital anomalies in the offspring of women with prepregnancy diabetes. Diabetes Care 2007, 30:1920-1925.

28. Ekbom P, Damm P, Feldt-Rasmussen B, et al.: Elevated thirdtrimester haemoglobin A1c predicts preterm delivery in type 1 diabetes. J Diabetes Complications 2008, 22:297-302.

29. Lapolla A, Dalfra MG, Bonomo M, et al.: Can plasma glucose and $\mathrm{HgAlc}$ predict fetal growth in mothers with different glucose tolerance levels? Diabetes Res Clin Pract 2007, 77:465-470.
30. Kernaghan D, Penney GC, Pearson DW: Birth weight and maternal glycated haemoglobin in pregnancies complicated by type 1 diabetes. Scott Med J 2007, 52:9-12.

31. Iafusco D, Stoppoloni F, Salvia G, et al.: Use of real time continuous glucose monitoring and intravenous insulin in type 1 diabetic mothers to prevent respiratory distress and hypoglycaemia in infants. BMC Pregnancy Childbirth 2008, 8:23.

32. Kestila KK, Ekblad UU, Ronnemaa T: Continuous glucose monitoring versus self-monitoring of blood glucose in the treatment of gestational diabetes mellitus. Diabetes Res Clin Pract 2007, 77:174-179.

33. McLachlan K, Jenkins A, O'Neal D: The role of continuous glucose monitoring in clinical decision-making in diabetes in pregnancy. Aust N Z J Obstet Gynaecol 2007, 47:186-190.

34. Murphy HR, Rayman G, Lewis K, et al.: Effectiveness of continuous glucose monitoring in pregnant women with diabetes: randomised clinical trial. BMJ 2008, 337:907-910.

35. Stenninger E, Lindqvist A, Aman J, et al.: Continuous subcutaneous glucose monitoring system in diabetic mothers during labor and postnatal glucose adaptation of their infants. Diabet Med 2008, 25:450-454.

36. Byrne EZ, Zisser HC, Jovanovic L: Continuous glucose monitoring: is it helpful in pregnancy? Curr Diabetes Rev 2008, 4:223-226.

37. Chitayat L, Zisser H, Jovanovic L: Continuous glucose monitoring during pregnancy. Diabetes Technol Ther 2009, 11:S105-S111.

38. - De Block C, Vertommen J, Manuel-y-Keenoy B, Van Gaal L: Minimally invasive and non-invasive continuous glucose monitoring systems: indications, advantages, limitations and clinical aspects. Curr Diabetes Rev 2008, 4:159-168. This is a comprehensive review of continuous glucose monitoring and the literature pertaining to it.

39. Block CD, Manuel-y-Keenoy B, Gaal LV: A review of current evidence with continuous glucose monitoring in patients with diabetes. J Diabetes Sci Technol 2008, 2:718-727.

40. Murphy HR: Integrating educational and technological interventions to improve pregnancy outcomes in women with diabetes. Diabetes Obes Metab 2010, 12:97-104.

41. Charlton MH: Commentary: The technology of continuous glucose monitoring. BMJ 2008, 337:911-914. 